\title{
A Review of Dynamic Rate-Versatile MIMO Mode Exchanging between Spatial Multiplexing and Assorted Qualities
}

Shiwani Dogra* and Navjot Kaur

School of Electronics and Electrical Engineering, Lovely Professional University, Phagwara - 144411, Punjab, India; dograshiwani98@gmail.com, navjot.12695@lpu.co.in

\begin{abstract}
MIMO gives high data rates and increase spectral efficiency through spatial diversity and multiplexing using STBC (Space Time Block Code) technique. STBC improve the reliability of wireless link. The main goal is to maximize the Average Spectral Efficiency (ASE) under the constraints that the A-BER should be lower. In Optimal method, A-BER approach, the objective is to boost the ASE under the requirement that the A-BER ought to be lower. In Sub optimal method, the SNR range appointed to a high request regulation builds, the ASE increments while the BER additionally increments in the same time. Better ASE (Area Spectral Efficiency) performance than the static MIMO mode switching scheme with adaptive modulation. In the traditional static mode, the MIMO mode change depending upon the normal SNR rather than the prompt channel condition. A rate-versatile modulation plan combined with mode exchanging between spatial multiplexing and orthogonal space-time piece coding. Initially discover which regulation level and mode amplify the phantom productivity with a given target bit blunder proportion (BER). In the event that the rates of the two MIMO modes are the same, select one mode that gives lower BER than the other. MIMO technology can be used in non-wireless communications systems. One example is the home networking standard ITU-T G.9963, which defines a power line communications system that uses MIMO techniques to transmit multiple signals over multiple AC wires.
\end{abstract}

Keywords: Assorted Qualities (Spatial Diversity), MIMO (Multiple Input Multiple Output), MIMO Switching Scheme, Spatial Multiplexing, Versatile

\section{Introduction}

MIMO remains for various info different yield and refers to the correspondence channel made with various transmit and get radio wires. The utilization of various reception apparatuses at the transmitter and collector in remote correspondence framework known as different inputs numerous yields 1 . MIMO is the same advancement that makes $4 \mathrm{G}$ and $802.11 \mathrm{n}$ so quick. It could likewise be called one receiving wire in and out. Most basically, it expands the quantity of reception apparatus at the transmitter and recipient. They work in show to enhance the gadgets execution by exchanging more information without requiring included data transfer capacity ${ }^{2}$. When the amplitude and period of a radio sign change quickly over a brief timeframe this Phenomenon is known as blurring. In remote correspondence channel the transmitter sign can make a trip from transmitter to beneficiary over numerous intelligent ways. For instance the transmitted signal from BTS may experience the ill effects of the structures adjacent before achieving the portable station ${ }^{3}$. Remote correspondence innovation has demonstrated that the utilization of numerous reception apparatuses at both transmitter and beneficiary sides enhances the likelihood of high information rates through multiplexing or to enhance execution through differing qualities contrasted with single radio wire frameworks. The framework with different radio wires at the transmitter and recipient is normally alluded is to as MIMO frameworks. This strategy offers higher ability to remote frameworks and

*Author for correspondence 
the limit increments straightly with the quantity of reception apparatuses and connection extra data transmission and power requirements. In radio, different information and numerous yield (MIMO), is the utilization of various receiving wires at both the transmitter and beneficiary to enhance correspondence execution. It is one of a few types of brilliant radio wire innovation. MIMO innovation has pulled in consideration in remote interchanges, since it offers noteworthy expansions in information throughput and connection range without extra data transmission or extended transmit power. MIMO is an essential piece of present day remote correspondence principles. A MIMO framework exploits the spatial differing qualities that are acquired by spatially isolated reception apparatuses in a thick multipath scrambling environment.

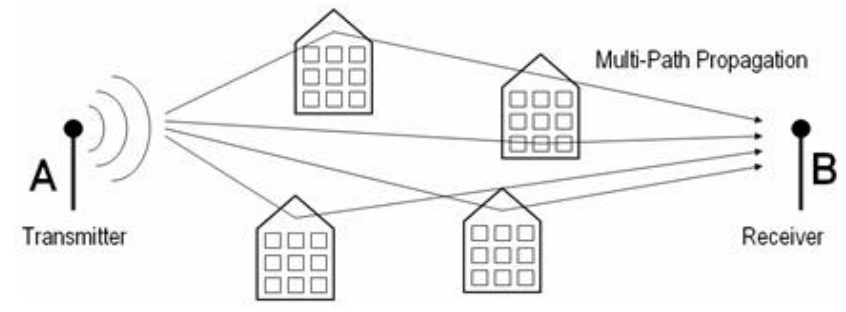

Figure 1. Diagram of a multipath propagation ${ }^{3}$.

Spatial multiplexing requires MIMO getting wire setup. In spatial multiplexing, a high rate signal is part into various lower rate streams and each stream is transmitted from a substitute transmit getting wire in the same repeat channel ${ }^{4}$. In case these signs land at the authority radio wire show with satisfactorily unmistakable spatial imprints and the recipient has exact CSI, it can separate these streams into parallel channels. Spatial multiplexing is an able technique for extending channel limit at higher sign to-confusion extents (SNR). The most great number of spatial streams is confined by the lesser of the amount of gathering devices at the transmitter or gatherer. Spatial multiplexing can be used without CSI at the transmitter, yet can be united with precoding if CSI is open ${ }^{5}$. Spatial multiplexing can in like manner be used for synchronous transmission to various recipients, known as space-division different access or multi-customer MIMO, in which case CSI is required at the transmitter. The arranging of recipients with different spatial imprints licenses incredible notice ability.

Reception apparatus assorted qualities (Antenna diversity) otherwise called space assorted qualities is any of a few remote differences plots that utilizations two or more receiving wires to enhance the quality and dependability of a remote connection ${ }^{6}$. Regularly, particularly in urban and indoor situations, there is no unmistakable viewable pathway (LOS) amongst transmitter and recipient. Rather the signal is reflected along numerous ways before at last being gotten. Each of these ricochets (a shot or hit that rebounds off a surface) can present stage shifts, time deferrals, weakening, and mutilations that can dangerously meddle with each other at the gap of the getting receiving wire. Radio wire assorted qualities is particularly successful at relieving these multipath circumstances. This is on the grounds that numerous reception apparatuses offer a collector a few perceptions of the same signal. Every reception apparatus will encounter an alternate obstruction environment. In this manner, in the event that one reception apparatus is encountering a profound blur, it is likely that another has an adequate signal. All in all such a signal can give a strong connection. While this is fundamentally seen in getting signals (differing qualities gathering), the simple has additionally demonstrated significant for transmitting signals (transmit assorted qualities) also ${ }^{\underline{z}}$. Intrinsically a reception apparatus assorted qualities plan requires extra equipment and coordination versus a solitary receiving wire signal however because of the shared characteristic of the signal ways a considerable lot of hardware can be shared. Likewise with the numerous signs there is a more prominent handling request put on the recipient, which can prompt more tightly plan prerequisites. Regularly, nonetheless, flag unwavering quality is foremost and utilizing numerous reception apparatuses is a viable approach to diminish the quantity of drop-outs and lost associations.

\section{Mimo System Description}

Multiple Input Multiple-Output (MIMO) remote radio wire frameworks have been perceived as a key innovation for future remote interchanges. In 1 portrays the effect of receiving wire determination on the execution of various info different yield (MIMO) frameworks over nonlinear correspondence channels. The creator have inferred careful logical expressions for assessing the PWEP execution of space-time trellis codes over nonlinear MIMO channel, instance of Rayleigh blurring, when radio wire determination is utilized at the collector side. Execution corruption because of nonlinearity in the channel diminishes as less quantities of receiving wires are chosen at the recipient, speaking to a few funds in SNR punishment 
because of nonlinearity for the lessened multifaceted nature framework. As future remote applications have made unquenchability as interest for- high information rate\| has exceedingly expanded. So it prompts the development of MIMO frameworks. The essential thought of MIMO is to enhance quality and information rate by utilizing numerous TX \& RX receiving wires ${ }^{1}$.

Multipath propagation has for quite some time been viewed as impairment on the grounds that it causes signal blurring. To moderate this issue, differing qualities systems were created. Radio wire differing qualities ${ }^{4}$ is an across the board type of assorted qualities. Late research has demonstrated that multipath spread can in certainty contribute to limit. Data hypothesis has demonstrated that with multipath spread, various radio wires at both transmitter and recipient can build up basically numerous parallel channels that work at the same time, on the same recurrence band at the same total radiated power. Multipath engendering has for quite some time been viewed as impairment on the grounds that it causes signal blurring. To moderate this issue, differing qualities systems were created. Radio wire differing qualities ${ }^{4}$ is an across the board type of assorted qualities. Late research has demonstrated that multipath spread can in certainty contribute to limit. Data hypothesis has demonstrated that with multipath spread, various radio wires at both transmitter and recipient can build up basically numerous parallel channels that work at the same time, on the same recurrence band at the same aggregate emanated power. Multipath causes multipath impedance including useful and ruinous obstruction, and stage moving of the signal. Dangerous impedance causes blurring. Where the sizes of the signs touching base by the different ways have an appropriation known as the Rayleigh circulation, this is known as Rayleigh blurring. Where one part (frequently, however not so much, a viewable pathway segment) commands, a Rician appropriation gives a more precise model, and this is known as Rician blurring.

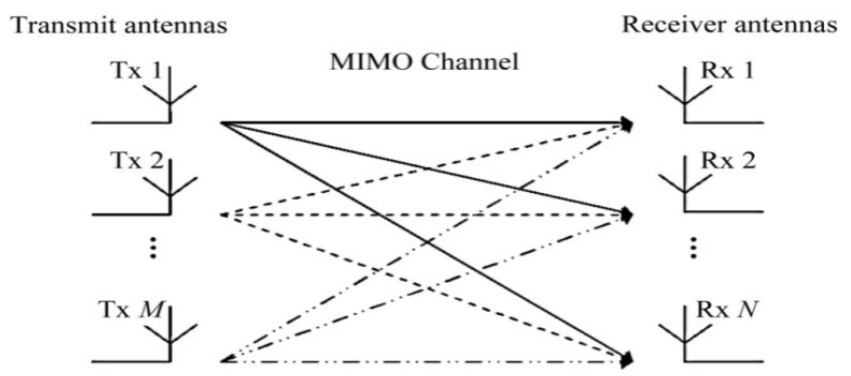

Figure 2. Diagram of a MIMO system²
MIMO technology has pulled in consideration in remote interchanges, since it offers noteworthy expansions in information throughput and connection range without extra transmission capacity or expanded transmit power. It accomplishes this objective by spreading the same aggregate transmit control over the reception apparatuses to accomplish a cluster pick up that enhances the unearthly proficiency (more bits every second per hertz of data transfer capacity) or to accomplish a differing qualities pick up that enhances the connection dependability (decreased blurring). As a result of these properties, MIMO is a critical piece of cutting edge remote correspondence models. Multi-radio wire frameworks can be ordered into three principle classes. Different reception apparatuses at the transmitter sides are typically material for pillar shaping purposes. Transmitter or beneficiary side various reception apparatuses for acknowledging distinctive (recurrence, space) assorted qualities plans. The second rate class incorporates frameworks with various transmitter and beneficiary reception apparatuses acknowledging spatial multiplexing as if there should arise an occurrence of MIMO system ${ }^{2}$. In the radio correspondences, MIMO implies various reception apparatuses on the both transmitter and recipient side of a particular radio connection. If there should arise an occurrence of spatial multiplexing diverse information images are transmitted on the radio connection by various receiving wires on the same recurrence inside the same time interim. Multipath proliferation is expected keeping in mind the end goal to guarantee the right operation of spatial multiplexing, since MIMO is performing better as far as direct limit in a rich dissipate multipath environment than if there should be an occurrence of environment with Line of Sight (LOS).

\subsection{Classification of MIMO Techniques}

MIMO procedures can be ordered as for the nature of channel learning at the transmitter and application zone.

\subsubsection{MIMO with Perfect Transmit Channel Knowledge}

In cases in which the transmitter knows about the channel, it can perform ideal consolidating techniques amid the force portion process. In that way, the SISO sub-channel that adds to the data exchange the most is supplied with more power. One strategy to compute the ideal force portion to the $\mathrm{n}$ sub-channels is to utilize the water pouring calculation. 


\subsubsection{MIMO with Partial Transmit Channel}

\section{Knowledge}

The misuse of the cluster increase may likewise be conceivable if the transmitter has just halfway channel information. Impeccable station information at the transmitter has been secured in past segment, however requires a high rate input join between the recipient and the transmitter to keep the last constantly educated about the station state. By complexity, misusing just the channel measurements or a quantized form of the channel at the transmitter requires a much lower rate input join. Precoding strategies for the most part comprise in joining a multi-mode beam former spreading the code words in orthogonal headings identified with the channel appropriation with a group of stars shaper, or all the more basically, a force designation plan. Radio wire determination procedures may depend just on halfway channel information, picking transmit or get reception apparatuses in view of the first and second-arrange insights of $\mathrm{H}$. Naturally, this comes to pick the radio wire sets with the most reduced relationship. Normally, such a procedure does not minimize the immediate mistake execution, but rather just the normal blunder rate. Thus, it drives for the most part to a coding increase and little differing qualities advantage.

\subsubsection{MIMO without Transmit Channel}

\section{Knowledge}

We know that the accomplished limit relies on upon the calculation utilized for assigning energy to every sub-channel. The hypothetical examination expects the channel state known at the beneficiary. This supposition stands right subsequent to the recipient as a rule performs following techniques with a specific end goal to acquire CSI, however the same thought does not have any significant bearing to the transmitter. At the point when the channel is not known at the transmitter, the transmitting signal is been measurably non-special, which infers that the nt parts of the transmitted signal are autonomous and equi-controlled at the transmit receiving wires. Thus, the force designated to each of the nt sub-channels is $\mathrm{pk}=\mathrm{p} / \mathrm{nt}$.

\subsection{MIMO Techniques based on Application}

\section{Area}

MIMO systems can be grouped taking into account the nature of channel learning at the transmitter as:

\subsubsection{Spatial Diversity in MIMO System}

MIMO framework use spatial differences to get critical channel gain, but it is not clear whether this choice is constantly superior to anything channel bouncing on two parallel channels when there is observable pathway. The utilization of various radio receiving wires to enhance signal respectability. S. Han et.al, talks about a high ghastly proficiency circulated receiving wire frameworks (DAS) which required vertical Bell Labs layered space-time. Microwave transmission frameworks in some cases utilize various spatial assorted get receiving wires, vertically isolated on a tower, for instance. As the probability is that sign won't endure the same level of lessening as it scatters somewhat and spreads along marginally diverse ways, the beneficiary with the most grounded sign expect control of the transmission. Remote LAN (WLAN) frameworks in light of the 802.11n detail utilize various transmit and get reception apparatuses in a method known as numerous information different yield (MIMO).

Code Division Different Access (CDMA) frameworks utilize a comparable methodology including rake recipients with multiple fingers. See also $802.11 \mathrm{n}$, antenna, attenuation, CDMA, scattering, MIMO, spread, rake collector, and time assorted qualities. Space assorted qualities has been a prevalent system in remote microwave correspondence. Space differing qualities at times called reception apparatus differences. it is normally actualized utilizing numerous radio wires or reception apparatus cluster orchestrate together in space for transmission and receiver. Multiple receiving wires are independently by an appropriate separation so that the individual signs are uncorrelated. The detachment necessities fluctuate with reception apparatus tallness, proliferation environment and recurrence. Commonly a partition of a couple of wavelengths is sufficient to get uncorrelated signs. In space diversity, the transmitted sign gave to the beneficiary as repetition in the space area. Time, frequency and space assorted qualities does not impel any misfortune in transmission capacity efficiency. In remote correspondence two or more customary differing qualities plans are consolidated to give multi dimensional differences.

\subsubsection{MIMO with Space Time Coding}

Space-Time Block Codes (STBCs) are the least complex sorts of spatial worldly codes that adventure the differing qualities offered in frameworks with a few transmit radio wires. In 1998, Alamouti ${ }^{4}$ planned a straightfor- 
ward transmission differences strategy for frameworks having two transmit reception apparatuses. This strategy gives full differences and requires straightforward direct operations at both transmission and gathering side. The encoding and deciphering procedures are performed with squares of transmission images. Alamouti's straightforward transmit assorted qualities plan was stretched out in and on account of the hypothesis of orthogonal outlines for numbers of transmit reception apparatuses. These codes are implied to in the written work as Orthogonal Space-Time Block Codes (OSTBCs).

\subsubsection{Alamouti Space Time Coding}

Space-time square coding is a procedure utilized as a part of remote correspondences to transmit numerous duplicates of an information stream over various reception apparatuses and to misuse the different got renditions of the information to enhance the unwavering quality of information exchange. The way that the transmitted sign must cross a possibly troublesome environment with disseminating, reflection, refraction thus on and may then be further tainted by warm clamor in the recipient implies that a portion of the got duplicates of the information will be "better" than others. This repetition results in a higher shot of having the capacity to utilize one or a greater amount of the got duplicates to effectively decipher the got signal. Truth be told, space-time coding consolidates every one of the duplicates of the got signal in an ideal approach to separate however much data from each of them as could be expected ${ }^{4}$.

\subsubsection{Spatial Multiplexing}

One of the key preferences of MIMO spatial multiplexing is the way that it can give extra information limit. MIMO spatial multiplexing accomplishes this by using the different ways and viably utilizing them as extra "channels" to convey information ${ }^{5}$. The most extreme measure of information that can be conveyed by a radio channel is restricted by the physical limits characterized under Shannon's Law. This is especially critical on the grounds that MIMO remote innovation gives a technique not of infringing upon the law, but rather expanding information rates past those conceivable on a solitary channel without its utilization. Spatial multiplexing offers a direct (in the quantity of transmit-get receiving wire sets or min (MR, $\mathrm{MT}$ ) increment in the transmission rate (or limit) for the same data transfer capacity and with no extra power use.
It is just conceivable in MIMO channels. Consider the instances of two transmit and two get radio wires. This can be reached out to more broad MIMO channels. The bit stream is part into two half-rate bit streams, adjusted and transmitted all the while from both the receiving wires. The collector, having complete information of the channel, recoups these individual piece streams and joins them to recuperate the first piece stream. Since the recipient knows about the channel it gives get differences, however the framework has no transmit assorted qualities since the bit streams are totally unique in relation to each other in that they convey entirely unexpected information. Accordingly spatial multiplexing expands the transmission rates relatively with the quantity of transmit-get radio wire sets.

\subsubsection{Beam forming MIMO Systems}

Beam forming is a critical sidekick to $4 \times 4$ Multiple Input Multiple Output (MIMO) innovations for Wi-Fi remote systems administration. When it is incorporated, it empowers emotional change in Wi-Fi 802.11ac/n execution, unwavering quality, reach and scope. The best sort of beam forming is rapid advanced beam forming. This kind of beam forming uses a progressed, on-chip computerized signal handling (DSP) calculation to increase complete control over Wi-Fi signals. By making a few autonomous sign ways to ideally centre radio vitality to and from customer gadgets on a for every bundle premise, execution is drastically made strides. On account of a two-stream arrangement, this makes it conceivable to guide the vitality of the radio wire cluster in the autonomous spatial bearings connected with both information streams, while at the same time maintaining a strategic distance from obstruction. $\mathrm{In}^{2}$ exhibited the execution examination of multi-client assorted qualities in multiuser transmits radio wire determination with maximal-proportion consolidating (TAS/MRC) system. When joined with $4 \times 4$ MIMO, dynamic advanced beam forming is especially capable. This is on account of a $4 \times 4$ MIMO framework bolsters two information streams and gives two additional transmit radio wires that might be utilized for beamforming, to permit critical centering of the vitality in two bearings while decreasing obstruction with coinciding frameworks. This sort of joined arrangement can convey from 12 to $25 \mathrm{~dB}$ of framework increases in respect to $802.11 \mathrm{ac} / \mathrm{n}$ remote LAN frameworks without element advanced transmit beam forming. The mix 
of $4 \mathrm{x} 4$ MIMO with element computerized beam forming likewise is versatile, which implies it can continually conform Wi-Fi execution in view of continuous occasions. At the end of the day, it ideally adjusts the transmit radio wire exhibit example to the spatial qualities of the MIMO channel recurrence reaction, furthermore to the quantity of information streams and collector positions. Accordingly, it can enhance fast execution and consistency over longer separations. Conversely, a 3x3 framework transmitting two information streams has altogether bring down dependability since there is one and only additional receiving wire to centre vitality in the two required headings. A $2 \times 2$ framework gets almost no advantage from transmit beam forming since has just the base required reception apparatuses. While dynamic computerized beamforming works with any number of get reception apparatuses, it is most effective in a $4 \times 4$ MIMO design.

\section{Spatial Diversity}

A high unearthly proficiency passed on radio wire framework (DAS) which required vertical Bell-Labs layered space-time. In any case, not at all like ordinary pointto-point various info numerous yield (MIMO) channels, DAS channels have diverse substantial scale blurring from different transmit receiving wires. The plan depends on huge scale blurring (shadow blurring and way misfortune) and is reasonable for VBLAST structures with zero-driving and progressive obstruction cancelation (ZF-SIC) collectors, guaranteeing adjusted normal image mistake rate (SER) execution in every layer. On the recipient side, an altered recognition request is utilized, which is acquired in the transmit receiving wire choice procedure. Re-enactment results demonstrate that the proposed plan gives great execution increases over equivalent power and rate transmission frameworks without radio wire choice ${ }^{8}$.Proposed a novel unitary downlink precoding plan for multiuser spatial multiplexing various information numerous yield (MIMO) frameworks. With the ideal CSI accessible at the transmitter and the direct decoder at the collector, the cost capacity was built in view of the base normal likelihood of vector image mistake and the outline technique for the precoding grids was given. These proposed precoding frameworks can totally wipe out co-channel obstruction for every client at the transmitter, and every client will in the end watch an impedance free single client channel, in this way stream- line the deciphering of every client. The effect of channel criticism blunders on the system execution and the upper limits of a few plans for execution examination have been researched ${ }^{5}$.Co-channel impedance presented by recurrence reuse under space division numerous entrances can be lessened by controlling the recurrence sharing as indicated by the clients spatial distinguishableness. The framework abstains from putting exceptionally associated clients in space into the same spatial multiplexing bunch ${ }^{9}$.

A heterogeneous optical access organizing situation, in which correspondence joins amongst source and destination hubs are heterogeneous, made out of free-space optical, Plastic Optical Fibre (POF), and indoor Infrared (IR) joins. A force variable rate-versatile low-thickness equality check (LDPC)- coded orthogonal recurrence division multiplexing (OFDM) plan is utilized to manage transfer speed impediments of POF and IR joins and the spatial differing qualities is utilized to manage environmental turbulence. The rate-versatile LDPC codes are planned utilizing a changed dynamic edge-development (MPEG) calculation. The outcomes exhibit a fast optical signal to an end-client with the proposed heterogeneous correspondence frameworks ${ }^{10}$. Time-division multiplexed exchanging (TOMS) based various info different yields (MIMO) channel sounders are generally utilized for remote channel estimations because of their powerful expenses. Nonetheless, estimation clamor, for example, stage commotion in the nearby oscillators and added substance white Gaussian clamor (AWGN) can bring about noteworthy mistakes in channel limit gauges ${ }^{\underline{11}}$. Wavelength-Division Multiplexing (WDM) has been the workhorse of information systems, obliging exponential activity development for two decades. As of late, in any case, progress in WDM limit research has especially backed off as investigations are firmly drawing closer major Shannon points of confinement of nonlinear fiber transmission. Space-Division Multiplexing (SDM) is relied upon to further scale system limits, utilizing parallel strands of single-mode fibre, uncoupled or coupled centres of multicore fibres, or even individual methods of few-mode fibre in blend with different information numerous yield (MIMO) computerized signal handling $\underline{12}$.

\section{Spatial Multiplexing}

Space-division numerous entrances (SDMA) in the downlink of multiuser different information, various yield (MIMO) remote correspondences system can give 
a considerable increase in framework throughput. The test in such multiuser frameworks is outlining transmits vectors while considering the co-channel obstruction of different clients. Normal advancement issues of interest incorporate the limit issue-boosting the aggregate data rate subject to a force imperative or the force control issue minimizing transmitted power such that a specific nature of-administration metric for every client is met. Neither of these issues has shut structure answers for the general multiuser MIMO channel, however the burden of specific requirements can prompt shut structure arrangements ${ }^{13}$. A Future cell system will utilize spatial multiplexing with various receiving wires at both the transmitter and recipient to exploit expansive limit picks up. In such frameworks it will be attractive to choose a subset of accessible transmit radio wires for connection instatement, support or handoff. Introduce a rule for selecting the ideal radio wire subset when direct, reasonable collectors are utilized over a gradually shifting channel. Utilization of the postpreparing SNRs of the multiplexed streams whereby the receiving wire subset that affects the biggest least SNR is picked. Reproductions show that our determination calculation additionally gives assorted qualities advantage accordingly making straight beneficiaries helpful over blurring channels ${ }^{14}$.

Different information various yield (MIMO) remote frameworks use reception apparatus clusters at both the transmitter and recipient to give correspondence joins considerable differences and limit. Spatial multiplexing is a typical space-time tweak strategy for MIMO correspondence frameworks where autonomous data streams are sent over various transmit reception apparatuses. Shockingly, spatial multiplexing is touchy to sick molding of the channel grid. Precoding can enhance the strength of spatial multiplexing to the detriment of full channel learning at the transmitter-which is regularly not practical. This correspondence proposes a quantized precoding framework where the ideal precoder is looked over a limited codebook known not beneficiary and transmitter $\frac{15}{}$. The transmit receiving wire choice issue for a zero compelling spatial multiplexing framework with information of the channel insights at the transmitter. We appear through Wish art network examination that the sign to-clamor proportion on the kth stream is a weighted Chi-squared variable with the weight equivalent to-the $k$ th corner to corner section of the reversed transmit relationship framework. We utilize this outcome to create determination calculations for two cases-amplifying ergodic limits and minimizing the normal likelihood of blunder. Monte Carlo recreations represent potential execution upgrades ${ }^{16}$. A multi-receiving wire transmission and gathering is generally touted as the key innovation for empowering remote broadband administrations, whose across the board achievement will require 10 times higher phantom effectiveness than current cell frameworks, at 10 times lower cost for every piece. Frightfully effective, modest cell frameworks are by definition thickly populated and obstruction constrained. In any case, spatial multiplexing MIMO frameworks whose central legitimacy is a gathered sensational increment in unearthly productivity lose quite a bit of their viability in abnormal amounts of obstruction. This article outlines a few ways to deal with taking care of obstruction in multicell MIMO frameworks. The examination is pertinent to any multi-receiving wire cell system. We contend that a large number of the conventional impedance administration strategies have constrained convenience when seen working together with $\mathrm{MIMO}^{17}$.

Spatial multiplexing frameworks in connected numerous information various yield (MIMO) blurring channels with equivalent force assigned to each transmit radio wire. Under this imperative, the number and subset of transmit receiving wires together with the transmit image heavenly bodies are resolved expecting information of the channel connection grids. We first consider a settled information rate framework and differ the quantity of transmit receiving wires and star grouping such that the base edge in the sign to-commotion proportion (SNR) is boosted for direct and Vertical Bell Laboratories Layered SpaceTime beneficiaries. We likewise infer transmit reception apparatus and star grouping determination criteria for a progressive obstruction cancelation recipient (SCR) with a settled discovery request and a variable number of bits transmitted on each substream. Contrasted and a framework utilizing every single accessible radio wire, execution results show huge additions utilizing a subset of transmit reception apparatuses, notwithstanding for free blurring channels. At last, we select a subset of transmit receiving wires to amplify information rate given a base SNR edge ${ }^{18}$. Precoder taking into account enhancement of the base Euclidean separation dmin between sign focuses at the collector side and for use in various info numerous yield (MIMO) spatial multiplexing frameworks. Accepting that channel state data (CSI) can be made accessible at the transmitter, the three stages, which are right now utilized as a part of examinations on MIMO 
frameworks, are performed. On account of this representation, an ideal dmin precoder is inferred on account of two diverse transmitted information streams. For quadrature stage shift keying (QPSK) adjustment, a numerical methodology demonstrates that the precoder outline relies on upon the channel qualities. Examinations with most extreme sign to-commotion proportion (SNR) technique and different precoders in view of criteria ${ }^{19}$. A two-information numerous yield (TIMO) framework speaks to an essential extraordinary instance of various info different yield (MIMO) frameworks in pragmatic situations. In this paper, transmit advancement for TIMO spatial multiplexing frameworks is examined. Least piece mistake rate (MBER) is utilized as the enhancement measure. Surmised MBER transmit power designation for an assortment of beneficiary structures is proposed, including zero-constraining, progressive obstruction cancelation (SIC), and requested SIC. Transmit-beam forming plans utilizing 4-ary beat abundancy adjustment and quaternary stage shift keying premixing are additionally proposed, which take out mistake floors in not well molded TIMO channels. It is demonstrated both diagnostically and by numerical recreations that the proposed plans offer better execution analyzed than existing plans ${ }^{20}$.

\section{Adaptive MIMO Mode Switching}

Versatile MIMO Multiple-info various yield (MIMO) systems can offer critical limit increases over conventional Single-Information Single Output (SISO) systems. Nonetheless, various radio wires require numerous RF chains which comprise of enhancers, simple to computerized converters, blenders, and so forth. That is ordinarily extremely costly. A methodology for decreasing the expense while keeping up the high potential information rate of a MIMO framework is to utilize a lessened number of RF chains at the collector and endeavour to ideally designate every chain to one of a bigger number of get reception apparatuses which are normally less expensive components. Along these lines, just the best arrangement of receiving wires is utilized, while the remaining radio wires are not utilized, in this way diminishing the quantity of required RF chains. As of late, a few calculations have been produced for selecting the ideal radio wire subset given a channel acknowledgment. In it is proposed to choose the subset of transmit or get receiving wires in view of the Shannon limit model. Radio wire choice calculations that minimize the bit mistake rate (BER) of direct collectors in spatial multiplexing systems are presented.
Antenna choice calculations are proposed to minimize the image blunder rate when orthogonal space-time piece coding is utilized as a part of MIMO frameworks. The fundamental issue in the calculations showed up in the writing is that they accept flawless channel learning to discover Berenguer is upheld by a Fulbright Fellowship. The ideal receiving wire is arranged. This paper presents discrete stochastic estimation calculations for selecting the ideal radio wire subset in light of cutting edge discrete stochastic enhancement methods found in the late operations research writing. These procedures improve a goal capacity over an arrangement of achievable parameters when the target capacity can't be assessed diagnostically however must be evaluated. The strategies are in the same soul as customary versatile sifting calculations, for example, the minimum mean-squares (LMS) calculation in which at every cycle, the calculation makes a move towards a superior arrangement. The MIMO framework model with receiving wire determination is displayed. We likewise detail the radio wire choice issue as a discrete stochastic enhancement issue. A general discrete stochastic streamlining calculation is exhibited and its joining properties are outlined. A few reception apparatus determination criteria are exhibited, including greatest limit, least bound on blunder rate and least mistake rate.

\section{Conclusion}

A dynamic rate-versatile MIMO mode exchanging plan between spatial multiplexing and differing qualities modes. The proposed dynamic plan indicates preferable ASE execution over the static MIMO mode exchanging plan with versatile tweak. In the routine static mode, the MIMO mode changes relying upon the normal SNR rather than the momentary channel condition. We likewise proposed an imperfect calculation to find the SNR edges with the ABER requirement in the high normal SNR range. At the point When the two MIMO modes have the same ISE, we can utilize another mode trading model considering BER example. The BER type strategy demonstrates the same execution as the Demmel condition number technique with lower computational unpredictability ${ }^{21}$.

\section{References}

1. Yang T-S, Hallen AD, Hallen H. Reliable adaptive modulation aided by observations of another fading channel. IEEE Transaction on Communication. 2004 Apr; 52(4):605-11. 
2. Kumar NS, Goldsmith AJ, Chua S-G. Adaptive coded modulation for fading channels. IEEE Transactions on Communications. 1998 May; 46(5):595-602.

3. Goldsmith A, Chua SG. Adaptive Coded Modulation for Fading Channels. Transactions on Communication. 1997 Jun; 3(2):1488-92.

4. Wang X. Queuing with adaptive modulation and coding over wireless links: Crosslayer analysis and design. IEEE Trans Wireless Communication. 2005 May; 4(5):1142-53.

5. Goldsmith AJ, Chua S-G. Adaptive coded modulation for fading channels. IEEE Trans Communication. 1998 May; 46(5):595-602.

6. Wang X, Tugnait JK. A bit-map-assisted dynamic queue protocol for multi-access wireless networks with multiple packet reception. IEEE Trans Signal Process. 2003 Aug; 51(3):2068-81.

7. Zheng H, Viswanathan H. Optimizing the ARQ performance in downlink packet data systems with scheduling. IEEE Trans Wireless Commun. 2005 Mar, 4(4):495-506.

8. Alouini, Goldsmith AJ. Adaptive modulation over Nakagami fading channels. Kluwer J Wireless Commun. 2000 May; 13(3):119-43.

9. Biglieri E, Caire G, Taricco G. Limiting performance of block fading channels with multiple antennas. IEEE Trans Inf Theory. 2001 May; 47(3):1273-89.

10. Sanghoi P, Kansal L. Analysis of WIMAX physical layer using spatial diversity. International Journal of Computer Application. 2012 May; 44(5):42-8.

11. Kansal L, Kansal A, Singh K. BER analysis of MIMOOFDM system using OSTBC code structure for M-PSK under different fading channels. International Journal of Scientific and Engineering Research. 2011 Nov; 2(11).

12. Sanghoi P, Kansal L. Analysis of WIMAX physical layer using spatial diversity under different fading channels. International Journal of Computer Application. 2012 May; 45(20).
13. Spencer QH, Swindiehurst AL. Zero-forcing methods for downlink spatial multiplexing in multiuser MIMO channels. IEEE Transactions on Signal Processing. 2004 Feb; 52(2):461-71.

14. Heath RW, Sandhu S, Paulraj ALA. Antenna selection for spatial multiplexing systems with linear receivers. IEEE Communication. 2001 May; 55(4):142-4.

15. Love DJ, Heath RW. Limited feedback unitary precoding for spatial multiplexing systems. IEEE Transactions on Information Theory. 2005 Aug; 51(8):2967-76.

16. Gore DA, Heath RW, Paulraj AJ. Transmit selection in spatial multiplexing systems. IEEE Communications. 2002 Nov; 6(11):491-3.

17. Andrews JG, Choi W, Heath JRRW. Overcoming interference in spatial multiplexing MIMO cellular networks. IEEE Wireless Communications. 2007 Dec; 14(6):95-104.

18. Narasimhan R. Spatial multiplexing with transmit antenna and constellation selection for correlated MIMO fading channels. IEEE Transaction on Signal Processing. 2003 Nov; 51(11):2829-38.

19. Collin L, Berder O, Rostaing P. Optimal minimum distance-based precoder for MIMO spatial multiplexing systems. IEEE Transaction on Signal Processing. 2004 Mar; 52(3):617-27.

20. Wang N, Blostein SD. Minimum BER transmit power allocation and beamforming for two input-multiple-output spatial multiplexing systems. IEEE Transaction on Vehicular Technology. 2004 Mar; 56(2):704-9.

21. Kasiselvanathan M, Kumar NS. BER performance analysis and comparison for large scale MIMO receiver. Indian Journal of Science and Technology. 2015 Dec; 8(35).

22. Ranjth S, Vishnupriya T. Analysis and design of SDF architecture for MIMO application. Indian Journal of Science and Technology. 2016 Feb; 9(8). 